\title{
Increased Phagocytosis of Platelets from Patients with Secondary Dengue Virus Infection by Human Macrophages
}

\author{
Shoko Honda, $\dagger$ Mariko Saito, $\dagger$ Efren M. Dimaano, Philip A. Morales, Maria T. G. Alonzo, \\ Lady-Anne C. Suarez, Natsuki Koike, Shingo Inoue, Atsushi Kumatori, \\ Ronald R. Matias, Filipinas F. Natividad, and Kazunori Oishi* \\ Department of Clinical Medicine and Virology, Institute of Tropical Medicine Nagasaki University, Japan; Department of \\ Virology, Graduate School of Medicine, Tohoku University, Japan; Department of Disaster Prevention System, Faculty of \\ Risk and Crisis Management, Chiba Institute of Science, Japan; Laboratory for Clinical Research on Infectious Diseases, \\ International Research Center for Infectious Diseases, Research Institute for Microbial Diseases, Osaka University, Japan; \\ Department of Blood Borne Diseases, San Lazaro Hospital, Manila, Philippines; Research and Biotechnology \\ Division, St. Luke's Medical Center, Quezon City, Philippines
}

\begin{abstract}
The relationship between the percent phagocytosis of platelets by differentiated THP-1 cells was examined using flowcytometry and the peripheral platelet counts as well as platelet-associated IgG (PAIgG) in 36 patients with secondary dengue virus (DV) infections. The percent phagocytosis and the levels of PAIgG were significantly increased in these patients during the acute phase compared with the healthy volunteers. The increased percent phagocytosis and PAIgG found during the acute phase significantly decreased during the convalescent phase. An inverse correlation between platelet count and the percent phagocytosis $(P=0.011)$ and the levels of PAIgG $(P=0.041)$ was found among these patients during the acute phase. No correlation was found, however, between the percent phagocytosis and the levels of PAIgG. Our present data suggest that accelerated platelet phagocytosis occurs during the acute phase of secondary DV infections, and it is one of the mechanisms of thrombocytopenia in this disease.
\end{abstract}

\section{INTRODUCTION}

Dengue virus (DV), a mosquito-borne human viral pathogen, belongs to the genus Flavivirus of the family Flaviviridae, and has four serotypes (DEN-1, DEN-2, DEN-3, and DEN-4). ${ }^{1}$ Dengue virus types 1-4 induce a wide spectrum of clinical manifestations, including hemorrhagic manifestations associated with thrombocytopenia and increased vascular permeability. ${ }^{2}$ Secondary DV infections, which are commonly observed in dengue-endemic areas, are more likely to constitute a risk factor for dengue hemorrhagic fever (DHF). ${ }^{3}$ The disease is now highly endemic in more than 100 tropical countries. The number of cases has rapidly increased during the past three decades, ${ }^{4}$ and it has become a major public health concern particularly in tropical and subtropical countries.

Although DV-induced bone marrow suppression decreases platelet synthesis, an immune mechanism of thrombocytopenia resulting in increased platelet destruction appears to be operative in patients with DHF. ${ }^{5}$ An increased level of plateletassociated $\operatorname{IgG}(\mathrm{PAIgG})$ is frequently observed in patients with chronic idiopathic thrombocytopenic purpura (ITP), but it also is found in a variety of other diseases. ${ }^{6.7}$ We previously demonstrated an inverse correlation between the levels of PAIgG and platelet count during the acute phase of secondary DV infections. ${ }^{8,9}$ We speculate that immune complexes of the DV with anti-DV IgG antibodies are located on the platelet, as a result of the direct binding of the DV to platelets. ${ }^{10}$ The results from our previous studies suggest that PAIgG formation, involving anti-DV IgG, may induce thrombocytopenia through both $\mathrm{Fc}$ receptor- and complement receptor-mediated platelet clearance by macrophages and/

*Address correspondence to Kazunori Oishi, Laboratory for Clinical Research on Infectious Diseases, International Research Center for Infectious Diseases, Research Institute for Microbial Diseases, Osaka University, 3-1, Yamadaoka, Japan. E-mail: oishik@biken.osaka-u.ac.jp $\dagger$ S. Honda and M. Saito equally contributed to the work described in this article. or complement-mediated platelet lysis. ${ }^{8}$ No studies, however, have been conducted to determine whether enhanced platelet phagocytosis by macrophages occurs in this disease. In this study, we establish an in vitro assay of platelet phagocytosis by macrophages using flowcytometry, and report an increased phagocytosis of platelets from patients with an acute phase of secondary DV infections.

\section{MATERIALS AND METHODS}

Patients and study design. Forty-two patients clinically suspected of having a DV infection were enrolled at San Lazaro Hospital between September 2006 and February 2007. Of these subjects, 40 were diagnosed with an acute phase of DV infection based on the results of a particle agglutination test for dengue $\operatorname{IgM}$ or reverse transcription-polymerase chain reaction (RT-PCR). ${ }^{11,12}$ Of these patients, 37 were diagnosed with an acute phase of a secondary DV infection based on the results of a hemagglutination inhibition (HI) test. ${ }^{13}$ Among the 37 patients with a secondary DV infection, we evaluated 36 patients who were examined for the peripheral platelet count, PAIgG levels, the frequency of platelet phagocytosis at the time of enrollment (acute phase), and 4 days after the first test (convalescent phase) in this study. One patient withdrew from the study following transferral to another hospital. Thirty-six healthy volunteers (HVs), who were age-matched, were also enrolled as control subjects at St. Luke's Medical Center during the same period. These HVs also received a particle agglutination test for dengue IgM, a platelet count, and an examination for PAIgG levels at the time of enrollment. Ethylenediaminetetraacetic acid (EDTA) and 3.8\% sodium citrate blood were drawn from these patients and from HVs for these tests. The platelet counts were determined using an automatic hemocytometer (Sysmex, Hyogo, Japan). The PAIgG levels were determined using a competitive enzyme-linked immunosorbent assay (ELISA), as previously described. ${ }^{9}$ The DHF was diagnosed by World Health Organization (WHO) criteria; a platelet count nadir of less than $100,000 / \mu \mathrm{L}$, 
hemorrhagic manifestations, and an increased hematocrit equal to or greater than $20 \%$ above the average or the presence of either pleural effusion or ascites fluid. ${ }^{14}$ Cases of DHF were further graded on a scale of I-IV. Dengue fever (DF) was defined as an increase in hematocrit of less than $20 \%$ and no detectable pleural effusion on the right lateral decubitus chest radiograph.

The research proposal for this study was approved by both the Bioethics Committees of San Lazaro Hospital and by St. Luke's Medical Center. Parents or guardians of all patients provided written informed consent. An interim target sample size of 62 was chosen to ensure that there would be at least a $70 \%$ chance for detecting a difference of $30 \%$ (50\% versus $20 \%$ ), with a one-sided alpha level of 0.05 , in the percent phagocytosis of platelets between patients with an acute phase of secondary DV infection and HVs.

Platelet preparation for platelet phagocytosis assay. Platelet-rich plasma (PRP) was separated from $5 \mathrm{~mL}$ of $3.8 \%$ sodium citrate blood drawn from patients with secondary DV infection and from HVs by centrifugation of $200 \times \mathrm{g}$ for $10 \mathrm{~min}$ at room temperature. After washing PRP with washing buffer [ $140 \mathrm{mM} \mathrm{NaCl}, 5 \mathrm{mM} \mathrm{KCl}, 12 \mathrm{mM}$ trisodium citrate, $10 \mathrm{mM}$ glucose, $12.5 \mathrm{mM}$ sucrose, and $1 \mu \mathrm{g} / \mathrm{mL} \mathrm{PGE}_{1}(\mathrm{pH} 6.0$ ) (Cayman Chemical, Ann Arbor, MI)], $2 \times 10^{8}$ washed platelets were suspended with $60 \mu \mathrm{L}$ of physiologic buffer (PB) $(140 \mathrm{mM}$ $\mathrm{NaCl}, 3 \mathrm{mM} \mathrm{KCl}, 0.5 \mathrm{mM} \mathrm{MgCl}_{2}, 5 \mathrm{mM} \mathrm{NaHCO}, 10 \mathrm{mM}$ glucose, and $10 \mathrm{mM}$ HEPES, [pH 7.4]) with or without $6.0 \mu \mathrm{g}$ of anti-human platelet monoclonal antibody (MAb) (mouse $\mathrm{IgG}_{1}$, Immuno-Biological Laboratories Co. Ltd, Takasaki, Japan) for $30 \mathrm{~min}$ at $37^{\circ} \mathrm{C}$, and washed with washing buffer. Washed platelets were then stained with $20 \mu \mathrm{M}$ of CellTracker Orange CMTMR (CTO; Molecular Probes, Inc., Eugene, $\mathrm{OR}$ ) for $30 \mathrm{~min}$ at $37^{\circ} \mathrm{C} .{ }^{15}$ The stained platelets were washed and suspended in $0.5 \mathrm{~mL}$ of $\mathrm{PB}$, and then incubated another $30 \mathrm{~min}$ at $37^{\circ} \mathrm{C}$ to remove excess dye. Washed platelets were resuspended in $0.7 \mathrm{~mL}$ of $\mathrm{PB}$, the number of the platelets were counted using an automatic hemocytometer. The efficiency of platelet labeling with CTO was determined to be $94.2 \%$ using flowcytometry (FACS Calibur, BD Biosciences, San Jose, CA). The frequency of platelet labeling with CTO was kept higher than $80 \%$ at least for 40 hours (data not shown). Anti-platelet $\mathrm{MAb}$ pretreated platelets from an $\mathrm{HV}$ were prepared in each platelet phagocytosis assay as a positive control in addition to untreated platelets from patients and HVs.

Differentiation of THP-1 cells. Undifferentiated human monocytic THP-1 cells (Cell number; JCRB-80194, Health Science Research Resources Bank, Japan) were cultured in RPMI-1640 medium with $25 \mathrm{mM}$ of HEPES, $10 \%$ fetal calf serum (FCS) (Hyclone, South Logan, UT), and penicillinstreptomycin (Gibco, Grandisland, NY), pH 7.2. The THP-1 cells were differentiated in the presence of $1 \mathrm{ng} / \mathrm{mL}$ of TGF- $\beta 1$ (Calbiochem, San Diego, CA) and $50 \mathrm{nM}$ of 1,25-(OH), vitamin $\mathrm{D}_{3}$ (Calbiochem) for 24 hours. ${ }^{16}$ Harvested THP-1 cells were washed twice by centrifugation, and the supernatant was removed. Twenty $\mu \mathrm{L}$ of phycoerythrin (PE)-conjugated mouse anti-human CD11b monoclonal $\mathrm{IgG}_{1}$ (BD Pharmingen, San Diego, CA) or PE-conjugated mouse monoclonal $\mathrm{IgG}_{1}$ isotype control (DakoCytomation, Glostrup, Denmark) were added to each tube and incubated at $4^{\circ} \mathrm{C}$ for $30 \mathrm{~min}$. Samples were washed twice and resuspended in PBS containing $1 \%$ paraformaldehyde, and then analyzed using flowcytometry. Although no expression of CD11b was found in the undifferentiated THP-1 cells, increased expression of CD11b was found in the differentiated THP-1 cells (data not shown). The expression of CD11b was specific because no increase was found in the fluorescent intensity of the differentiated cells stained with PE-conjugated control antibody.

In vitro platelet phagocytosis. Differentiated THP-1 cells were seeded at $1 \times 10^{6}$ cells/well using a 24-well plate in Hanks' balanced salt solution containing $0.15 \mathrm{mM} \mathrm{CaCl}_{2}$ and $1.0 \mathrm{mM}$ $\mathrm{MgCl}_{2}$ (HBSS $^{++}$, Nissui, Tokyo, Japan) $\mathrm{pH} 7.2$, for 1 hour to adhere to the cells. Fifteen nM of phorbol-12-myristate-13acetate (PMA) (Sigma, St. Louis, MO) were added after incubation for another hour to activate the THP-1 cells. ${ }^{15}$ The $5 \times$ $10^{6}$ platelets labeled with CTO were added to each well and the plates were centrifuged at $500 \times \mathrm{g}$ for $5 \mathrm{~min}$ at room temperature, with incubation at $37^{\circ} \mathrm{C}$ for $30 \mathrm{~min}$. After incubation, the adherent cells were washed twice using cold $5 \mathrm{mM}$ EDTA-PBS, the cells were then detached using $0.05 \%$ trypsin- $0.53 \mathrm{mM}$ EDTA for $5 \mathrm{~min}$ at $37^{\circ} \mathrm{C}$. After stopping the reaction by adding RPMI-1640 with $10 \%$ FCS, detached cells were separated by strong pipeting on ice, they were then collected by centrifugation at $200 \times \mathrm{g}$ for $5 \mathrm{~min}$ at $4^{\circ} \mathrm{C}$. Recovered cells were stained with fluorescein isothiocyanate (FITC) conjugated anti-human CD61 mouse IgG (DakoCytomation) for $30 \mathrm{~min}$ at $4{ }^{\circ} \mathrm{C}$. Cells were washed twice and resuspended in PBS containing $1 \%$ paraformaldehyde, followed by analysis using flowcytometry. In preliminary experiments, a negligible fluorescent signal of CTO was detected in the supernatants of washing buffer. However, no direct staining of differentiated THP-1 cells with CTO in the supernatants of wash buffer for CTO-stained platelets was confirmed by flowcytometry (data not shown).

Flowcytometry analysis. The frequency of platelet phagocytosis by differentiated THP-1 cells was determined by the frequency of CTO positive and platelet-specific marker CD61 negative cells. The differentiated THP-1 cells were gated and 10,000 events were acquired from each sample. For the standardization of the values for platelet phagocytosis, the percent phagocytosis was expressed using the following formula: the frequency of phagocytosis of test platelets divided by the frequency of phagocytosis of the positive control platelets (pretreated with anti-platelet MAb) $\times 100$.

Statistical analysis. All data were expressed as the mean \pm SD. Platelet counts and PAIgG levels during the period between the acute and convalescent phase were tested using a Wilcoxon signed rank test. The levels of platelet phagocytosis, PAIgG, platelet count between HVs and patients with DV infections, and platelet count between patients with DF and DHF were analyzed using the Mann-Whitney $U$ test. The significance of the correlations was estimated using the Pearson correlation; $P<0.05$ was considered to be significant. The SPSS statistical software, version 13.0 (SPSS Inc., Chicago, IL) was used for data analysis.

\section{RESULTS}

Of the 36 patients with secondary DV infections, 24 and 12 were diagnosed as DF and DHF, respectively (Table 1). Twelve patients with DHF were further classified into DHF I $(N=2)$ and DHF II $(N=10)$. No cases of DHF III or IV were included. The peripheral platelet counts were significantly lower in patients with DV infection than those in HVs $(P<0.05)$. Although the platelet counts and the increase in the 
TABLE 1

Laboratory data on patients with acute phase of secondary dengue virus infection and healthy volunteers

\begin{tabular}{lcccccc}
\hline \multicolumn{1}{c}{ Diagnosis (n) } & Age (years) & Days after onset & \% Increase in hematocrit & Platelet count $\left(\times 10^{3 / \mu L}\right)$ & PAIgG value (ng/10 PLT) & Percent phagocytosis \\
\hline HV (36) & $20.1 \pm 7.1$ & - & - & $322.7 \pm 98.1$ & $11.4 \pm 10.1$ & $23.5 \pm 23.0$ \\
DV infection (36) & $20.2 \pm 5.7$ & $5.5 \pm 0.7$ & $20.3 \pm 11.7$ & $53.1 \pm 28.0^{*}$ & $47.5 \pm 70.7 *$ & $55.5 \pm 60.4^{*}$ \\
DF (24) & $20.1 \pm 5.3$ & $5.4 \pm 0.7$ & $10.1 \pm 4.9$ & $60.0 \pm 24.3$ & $34.5 \pm 47.8$ & $47.7 \pm 52.8$ \\
DHF (12) & $20.3 \pm 6.8$ & $5.7 \pm 0.5$ & $29.9 \pm 9.9^{* *}$ & $39.3 \pm 30.8^{* *}$ & $71.5 \pm 98.4$ & $71.8 \pm 73.2$ \\
\hline
\end{tabular}

$\mathrm{PLT}=$ platelets $\mathrm{HV}=$ healthy volunteer $\mathrm{DV}=$ dengue virus; $\mathrm{DF}=$ dengue fever; $\mathrm{DHF}=$ dengue hemorrhagic fever.

$* P<0.05$ (versus $\mathrm{HV}$ ), ${ }^{* *} P<0.05$ (versus $\mathrm{DF}$ ).

hematocrit were significantly different in patients with DHF from patients with DF $(P<0.05)$, no significant difference was found between those two subgroups with respect to the demographic data, which includes age and days after onset. The levels of PAIgG were significantly higher in patients with DV infection than those in HVs $(P<0.05)$. Although the levels of PAIgG were higher in DHF patients than those in DF patients, no significant difference was found between those two subgroups, which is in disagreement with our previous report. ${ }^{9}$

Representative data of the frequency of platelet phagocytosis in a single experiment are shown in Figure 1. The frequencies of phagocytosis were $20.8 \%$ for platelets from an HV that were pretreated with anti-platelet MAb (Figure 1A), 3.2\% for untreated platelet from an HV (Figure 1B), and 16.8\% for untreated platelets from a patient with DF (Figure 1C). The values of percent phagocytosis were $15.4 \%$ for an $\mathrm{HV}$ and $80.8 \%$ for the patient with DF, respectively. The percent phagocytosis was significantly higher in the total number of patients with secondary DV infections than in the total number of HVs $(P<0.05$, Table 1$)$. The percent phagocytosis was similarly higher in DHF patients than in DF patients, but no significant difference was found between those two subgroups.

Between the acute and convalescent phases, the changes in platelet counts and percent platelet phagocytosis or PAIgG were compared in 36 patients with a secondary DV infection in the period between the acute and convalescent phases. The low baseline of platelet counts during the acute phase (53.1 $\left.\pm 28.0 \times 10^{3} / \mu \mathrm{L}\right)$ significantly increased and recovered to a normal range during the convalescent phase $(374.4 \pm 92.2$ $\times 10^{3} / \mu \mathrm{L} ; P<0.01$, Figure $\left.2 \mathrm{~A}\right)$ in these patients. In contrast, the increased baseline PAIgG $\left(47.5 \pm 70.7 \mathrm{ng} / 10^{7}\right.$ platelets $)$ and percent phagocytosis $(55.5 \pm 60.4 \%)$ decreased significantly during the acute phase, and returned to a normal level $\left(21.0 \pm 17.6 \mathrm{ng} / 10^{7}\right.$ platelets for PAIgG, $24.5 \pm 34.6 \%$ for the percent phagocytosis) during the convalescent phase in these subjects $(P<0.05$ for PAIgG; Figure $2 \mathrm{~B}, P<0.05$ for the percent phagocytosis; Figure $2 \mathrm{C}$ ). A significant inverse correlation was confirmed between the platelet count and the PAIgG level among the total 36 patients during the acute phase of secondary DV infection (Figure 3A), which is consistent with our previous results. ${ }^{8,9}$ A significant inverse correlation was also found between the platelet count and the percent phagocytosis (Figure 3B) among these subjects. On the other hand, no significant correlation was found between the percent phagocytosis and the levels of PAIgG among these patients (Figure 3C).

\section{DISCUSSION}

In this study, we demonstrated that the percent phagocytosis of platelets from patients during the acute phase of secondary DV infection was significantly increased, compared with those from healthy volunteers, using an in vitro assay. The percent phagocytosis of platelets was significantly inversely correlated with platelet count during the acute phase among these patients, although no significant correlation was found between the percent phagocytosis of platelets and PAIgG levels. Because we previously detected anti-DV IgG and DV RNA on the platelets from patients with secondary DV infections, but not from healthy volunteers, the presence of immune complexes on the platelets may contribute to the increased phagocytosis of platelets among patients with an acute phase of secondary DV infection..$^{8,9}$ Although no correlation between the levels of PAIgG and platelet phagocytosis was found in this study, the values of PAIgG, which were determined by a competitive ELISA using anti-human IgG, may not reflect the amount of anti-DV IgG on the platelets in each individual case. A correlation between the levels of platelet-associated
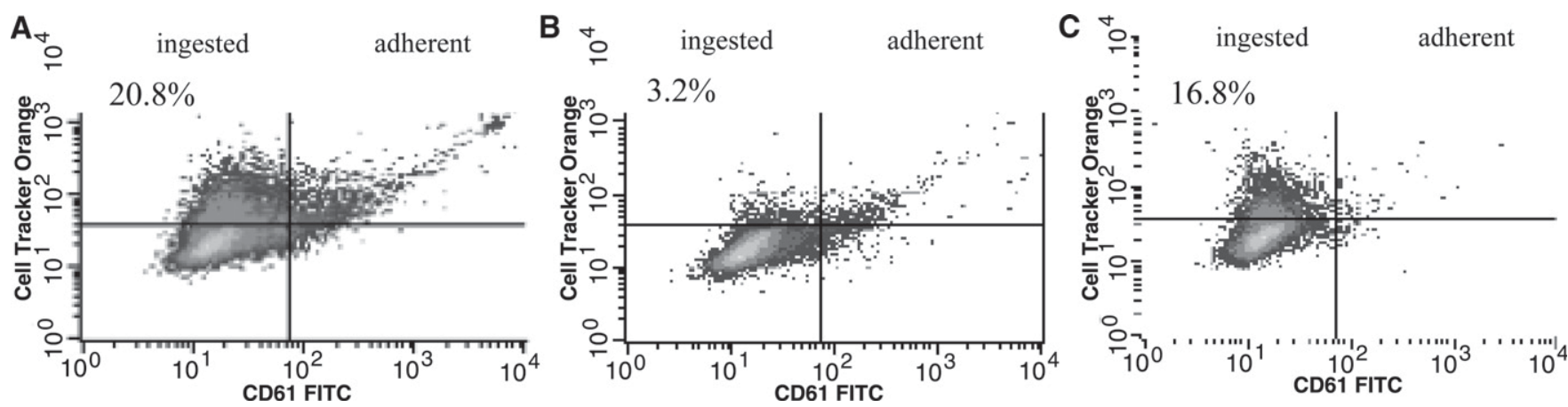

FIGURE 1. The frequencies of phagocytosis of platelets from a healthy volunteer (HV) with (A) or without anti-platelet monoclonal antibody treatment $(\mathbf{B})$, or untreated platelets from a patient with dengue fever (DF) $(\mathbf{C})$ are shown. The CellTracker Orange CMTMR (CTO) positive and CD61 negative cells (upper-left region) were considered to be the differentiated THP-1 cells that ingested platelets. The values of percent phagocytosis were determined to be $15.4 \%$ for an $\mathrm{HV}$ and $80.8 \%$ for a patient with DF according to the formula described in the Materials and Methods. 

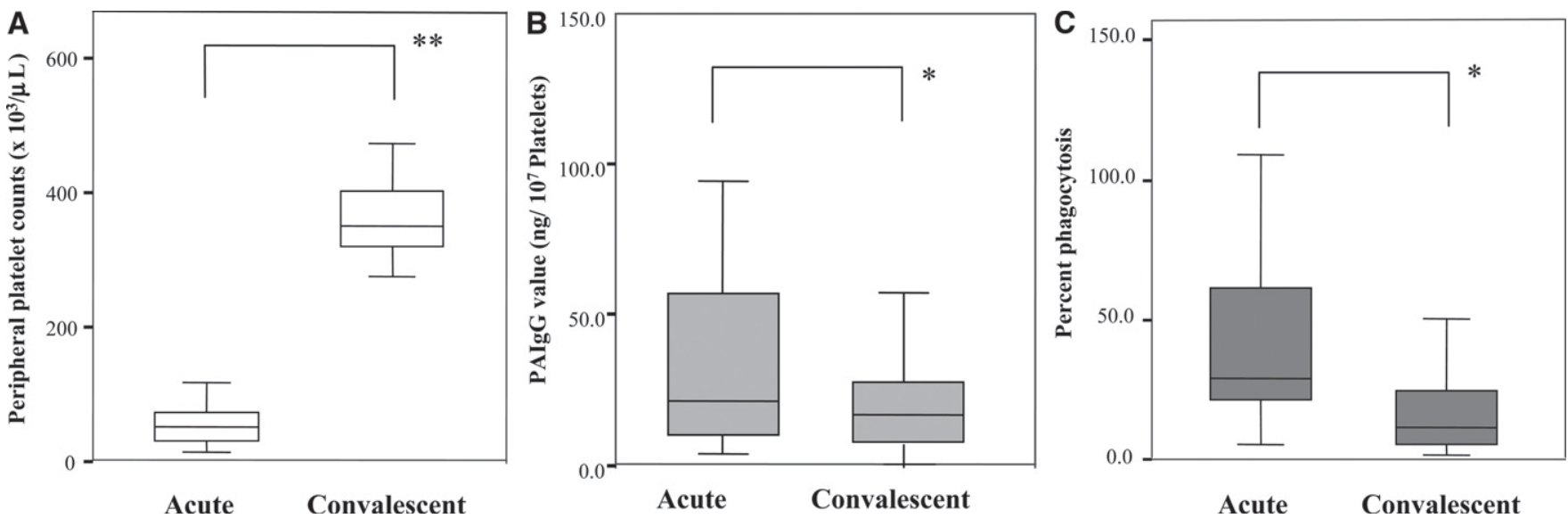

Figure 2. Comparisons of the peripheral platelet count $(\mathbf{A} ; N=36), \operatorname{PAIgG}(\mathbf{B} ; N=34)$, and the percent phagocytosis $(\mathbf{C} ; N=36)$ between the acute (the first test) and convalescent phase (four days after the first test) of secondary dengue virus infections. $* P<0.05, * * P<0.01$.

anti-DV IgG and the percent phagocytosis should be examined, although the assay for platelet-associated anti-DV IgG is not currently available.

Mitrakul and others ${ }^{17}$ reported that platelet survival was shortened in patients with an acute phase of DHF using radiolabeled platelets more than 30 years ago. They suggested that the damaged platelets were being trapped and sequestered in the liver rather than in the spleen, as is usually the case in this disease. Their finding of platelet clearance in the livers of patients with an acute phase of DHF could be explained, in part, by an increased phagocytosis of platelets as shown by the results of the present study.

We previously reported a lack of efficacy of a high dose of intravenous immunoglobulin for patients with secondary DV infection, and suggested that platelet clearance by macrophages through $\mathrm{Fc} \gamma$ receptors was not a primary mechanism in this disease.$^{18}$ Furthermore, in a preliminary experiment on an inhibition assay of phagocytosis of platelets from a patient of DF, a partial inhibition of platelet phagocytosis by differentiated THP-1 cells was found with mouse anti-human complement receptor 3 (CR3) (CD11b) MAb, compared with control MAb. Because complement activation mediated by circulating viral antigen is involved in the pathogenesis of this disease, ${ }^{19,20}$ platelet clearance by macrophages through CR3 may be involved in this disease. Further studies of inhibition assays are required to draw conclusions for the precise molecular mechanisms of platelet phagocytosis in secondary DV infections.

Platelets, anucleated blood cells, may undergo an apoptotic program. Treatment of platelets with a variety of platelet agonists induces apoptosis and caspases, which are key effectors of apoptosis, are involved in this in vitro phenomenon. ${ }^{21,22}$ Brown and others ${ }^{23}$ reported an increased expression of proapoptotic proteins by flowcytometry and morphologic changes similar to those of granulocyte apoptosis found by electron microscopy in aged platelets. Another possible mechanism for the increased phagocytosis of platelets from patients in this study could be the scavenger receptor-mediated phagocytosis.

In conclusion, this study demonstrated an increased phagocytosis of platelets freshly isolated from patients during the acute phase of secondary DV infection in an in vitro assay employing differentiated THP-1 cells. Increased platelet phagocytosis was significantly associated with thrombocytopenia during the acute phase of this disease. Further studies are

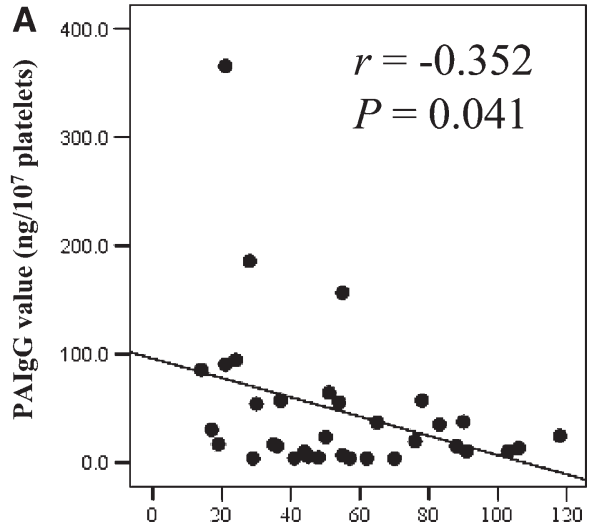

Peripheral platelet count $\left(10^{3} / \mu \mathrm{L}\right)$
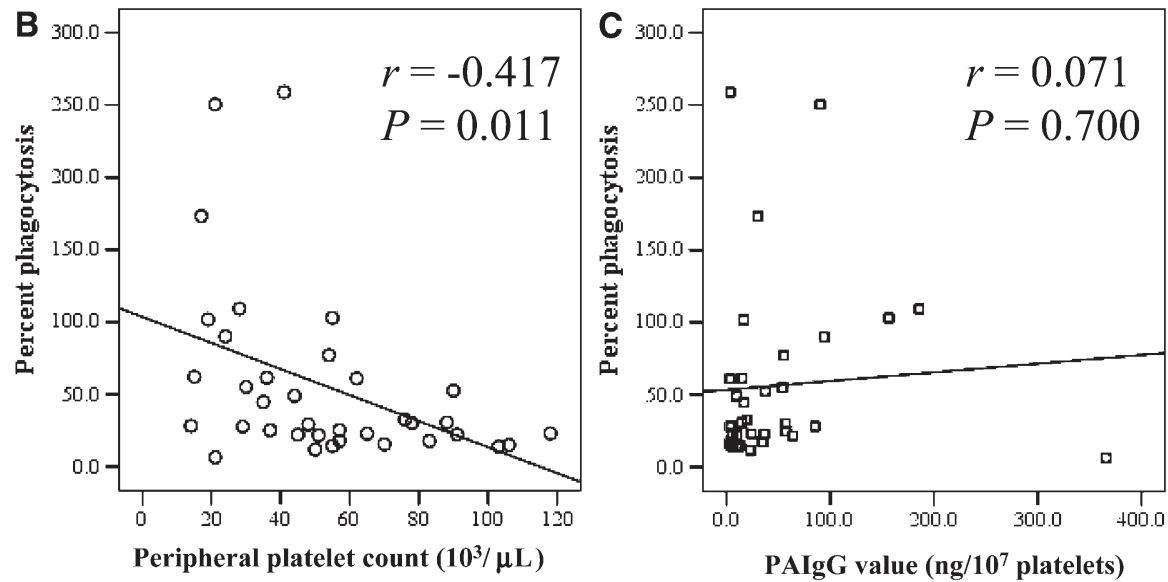

FIGURE 3. Relationship between the peripheral platelet count and either the levels of PAIgG $(\mathbf{A} ; N=34$ : closed circles $)$ or the percent phagocytosis $(\mathbf{B} ; N=36$ : open circles) and the relationship between the levels of PAIgG and the percent phagocytosis $(\mathbf{C} ; N=34$ : open squares $)$ in patients with an acute phase of a secondary dengue virus infection. 
required to determine the molecular mechanisms of platelet phagocytosis by macrophages in a secondary DV infection.

Received July 9, 2008. Accepted for publication January 16, 2009.

Acknowledgments: We thank the staff of San Lazaro Hospital and the Research Biotechnology Division, St. Luke's Medical Center.

Financial support: This study was supported by a Grant-in-Aid for Scientific Research (B: 16406029) from the Ministry of Education, Science and Culture, Japan and the 21st Century Center of Excellence (COE) Program of Nagasaki University.

Authors' addresses: Shoko Honda and Shingo Inoue, Department of Internal Medicine and Virology, Institute of Tropical Medicine Nagasaki University, 1-12-4 Sakamoto, Nagasaki 852-8523, Japan. Mariko Saito, Department of Virology, Graduate School of Medicine, Tohoku University, 2-1 Seiryo-machi, Aoba-ku, Sendai 980-8575, Japan. Efren M. Dimaano and Philip A. Morales, Blood Borne Diseases, San Lazaro Hospital, Manila, Philippines. Maria T. G. Alonzo, Ronald R. Matias, and Filipinas F. Natividad, Research and Biotechnology Division, St. Luke's Medical Center, 279 E. Rodriguez Sr. Boulevard, Cathedral Heights, Quezon City, Philippines 1102. Lady-Anne C. Suarez, E-mail: suarezlacs@yahoo.com. Natsuki Koike, E-mail: nakkey2@yahoo.co.jp. Atsushi Kumatori, Faculty of Risk and Crisis Management, Chiba Institute of Science, Choshi 2880025, Japan. Kazunori Oishi, Laboratory for Clinical Research on Infectious Diseases, International Research Center for Infectious Diseases, Research Institute for Microbial Diseases, Osaka University, 3-1 Yamadaoka, Suita, Osaka 565-0871, Japan, Tel: +81-6-6879-4253, Fax: +81-6-6879-4255, E-mail: oishik@biken.osaka-u.ac.jp.

Reprint requests: Kazunori Oishi, Laboratory for Clinical Research on Infectious Diseases, International Research Center for Infectious Diseases, Research Institute for Microbial Diseases, Osaka University, 3-1 Yamadaoka, Osaka 565-0871, Japan, Tel: +81-6-6879-4253, Fax: +81-6-6879-4255, E-mail: oishik@biken.osaka-u.ac.jp.

\section{REFERENCES}

1. Gubler DJ, 1998. Dengue and dengue hemorrhagic fever. Clin Microbiol Rev 11: 480-496.

2. Oishi K, Saito M, Mapua CA, Natividad FF, 2007. Dengue illness: clinical features and pathogenesis. I Infect Chemother 13: $125-133$.

3. Halstead SB, 1988. Pathogenesis of dengue: challenges of molecular biology. Science 239: 476-481.

4. Gubler DJ,2002. Epidemic dengue/dengue hemorrhagic fever as a public health, social and economic problems in the 21 st century. Trends Microbiol 10: 100-103.

5. La Russa VF, Innis BL, 1995. Mechanism of dengue virus-induced bone marrow suppression. Baillieres Clin Haematol 8: 249-270.

6. Cines DB, Blanchette VS, Chir B, 2002. Immune thrombocytopenic purpura. N Engl J Med 346: 995-1007.

7. Rand ML, Wright JF, 1998. Virus-associated idiopathic thrombocytopenic purpura. Transfus Sci 19: 253-259.

8. Oishi K, Inoue S, Cinco MT, Dimaano EM, Alera MT, Alfon JA, Abanes F, Cruz DJ, Matias RR, Matsuura H, Hasebe F, Tanimura S, Kumatori A, Morita K, Natividad FF, Nagatake T, 2003. Correlation between increased platelet-associated $\mathrm{IgG}$ and thrombocytopenia in secondary dengue virus infections. $J$ Med Virol 71: 259-264.
9. Saito M, Oishi K, Inoue S, Dimaano EM, Alera MTP, Robles MP, Estrella JR, Kumatori A, Moji K, Alonzo BMT, Buerano CC, Matias RR, Morita K, Natividad FF, Nagatake T, 2004. Association of increased platelet-associated immunoglobulins with thrombocytopenia and the severity of disease in secondary dengue virus infections. Clin Exp Immunol 138: 299-303.

10. Wang S, He R, Patarapotikul J, Innis BL, Anderson R, 1995. Antibody-enhanced binding of dengue-2 virus to human platelet. Virology 213: 254-257.

11. Berlioz-Arthaud A, Marfel M, Durand AM, Ogawa T, 2005. Evaluation of a new anti-dengue virus IgM particle agglutination kit in the context of the Pacific Islands. WHO Dengue Bulletin 29: 70-78.

12. Morita K, Tanaka M, Igarashi A, 1991. Rapid identification of dengue virus serotypes by using polymerase chain reaction. J Clin Microbiol 29: 2107-2110.

13. Clarke DH, Casals J, 1958. Techniques for hemagglutination and hemagglutination-inhibition with arthropod-borne viruses. Am J Trop Med Hyg 7: 561-573.

14. World Health Organization, 1997. Dengue Haemorrhagic Fever: Diagnosis, Treatment, Prevention and Control (Second edition). Geneva, Switzerland: WHO.

15. Hoffmeister KM, Felbinger TW, Falet H, Denis CV, Bergmeier W, Nayadas TN, von Andrian UH, Wagner DD, Stossel TP, Hartwig $\mathrm{JH}, 2003$. The clearance mechanism of chilled blood platelets. Cell 112: 87-97.

16. Simon DI, Chen Z, Xu H, Li CQ, Dong J-F, McIntire LV, Ballantyne CM, Zhang L, Furman MI, Berndt MC, López JA, 2000. Platelet glycoprotein $\mathrm{Ib} \alpha$ is a counterreceptor for leukocyte integrin Mac-1(CD11b/CD18). J Exp Med 192: 193-204.

17. Mitrakul C, Poshyachinda M, Futralul P, Sangkawibha N, Ahandrik S, 1977. Hemostatic and platelet kinetic studies in dengue hemorrhagic fever. Am J Trop Med Hyg 26: 975-984.

18. Bokisch VA, Top FH Jr, Russell PK, Dixon FJ, Muller-Eberhard HJ, 1973. The potential pathogenic role of complement in dengue hemorrhagic shock syndrome. $N$ Engl J Med 289: 996-1000.

19. Avirutnan P, Punyanadee N, Noisaran S, Komoltri C, Thiemmeca S, Auethavornanan K, Jairungsri A, Kanlaya R, Tangtawornchailul N, Puttikhunt C, Pattnakisakul S, Yenchitsomnus P, Mongkolsapaya J, Kasinrerk W, Sittisombut N, Husmann M, Blettner M, Vasanawathana S, Bhakdi S, Mlasit P, 2006. Vascular leakage in severe dengue virus infections: a potential role for the nonstructural viral protein NS1 and complement. J Infect Dis 193: 1078-1088.

20. Dimaano E, Saito M, Honda S, Miranda EA, Alonzo MT, Valerio MD, Mapua CD, Inoue S, Kumatori A, Matias R, Natividad FF, Oishi K, 2007. Lack of efficacy of high dose intravenous immunoglobulin treatment of severe thrombocytopenia in patients with secondary dengue virus infection. Am J Trop Med Hyg 77: 1135-1138.

21. Shcherbina A, Remold-O'Donnell E, 1999. Role of caspase in a subset of human platelet activation responses. Blood 93: 4222-4231.

22. Tonon G, Luo X, Greco NJ, Chen W, Shi Y, Jamieson GA, 2003. Weak platelet agonists and U46619 induces apoptosis-like events in the absence of phosphatidylserine exposure. Thromb Res 107: 345-350.

23. Brown SB, Clarke MCH, Magowan L, Sanderson H, Savill J, 2000. Constitutive death of platelets leading to scavenger receptormediated phagocytosis. J Biol Chem 275: 5987-5996. 